endesigns eine weitere Selektion berücksichtigt werden: In der SCORPION-Studie wurde die Operabilität anhand einer diagnostischen Laparoskopie beurteilt. Bei 15

\section{"Die Ergebnisse sind nur schwer in Therapieempfehlungen für unsere Patientinnen zu überführen."}

der 240 Patientinnen (6,25\%) war die diagnostische Laparoskopie technisch wegen großer Tumormassen mit fehlender Einsicht in das Abdomen nicht möglich. Wie mit diesen Befunden umzugehen ist, wird im vorliegenden Manuskript nicht thematisiert. Bisher wird die Beurteilung der Operabilität mithilfe einer diagnostischen Laparoskopie allgemein als nicht zuverlässig genug angesehen.

Deshalb ist dieser Eingriff bisher auch nicht in internationalen Leitlinien verankert. Daher sind auch die Ergebnisse dieser Studie nur schwer in Behandlungsempfehlungen für unsere Patientinnen mit fortgeschritte- nem Ovarialkarzinom überführbar. Um die Frage der optimalen Behandlungssequenz mit höchster Evidenz und möglichst wenigen Einflussfaktoren zu untersuchen, wurde kürzlich unter Federführung der AGO-Studiengruppe Ovar mit der AGO-OP.6-Studie (TRUST $=$ Trial on Radical Upfront Surgical Therapy) ein internationales Studienkonzept initiiert, bei dem mit AGO-OVAR19 auch ein besonderer Fokus auf Morbidität und Lebensqualität liegt. In der TRUSTStudie werden Patientinnen mit Verdacht auf ein fortgeschrittenes EOC prospektiv 1:1 randomisiert.

Alle Patientinnen werden registriert und diejenigen eingeschlossen, bei denen das Ovarialkarzinom nach Einschätzung eines erfahrenen Operateurs potenziell komplett resektabel ist (denn das stellt den entscheidenden Faktor der Operation dar). Je nach Randomisation werden die Patientinnen dann entweder primär oder nach histologischer Sicherung und 3 Zyklen neoadjuvanter Chemotherapie im Intervall mit dem Ziel der makroskopischen Tumorfreiheit ope- riert (Details unter www.ago-online.de). Außerhalb von Studien gilt weiterhin der Standard, Patientinnen mit fortgeschrittenem Ovarialkarzinom mit primärem Debulking, gefolgt von Chemotherapie und ggf. antiangiogener Therapie, zu behandeln.

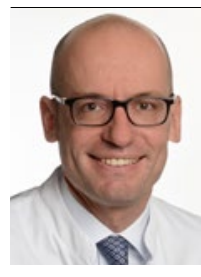

Prof. Dr. med. Sven Mahner Klinik und Poliklinik für Frauenheilkunde und Geburtshilfe, Klinikum der Universität München Sven.Mahner@med. uni-muenchen.de

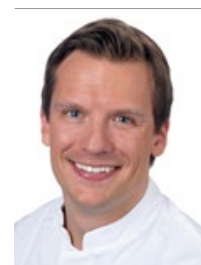

Dr. med. Fabian Trillsch Klinik und Poliklinik für Frauenheilkunde und Geburtshilfe, Klinikum der Universität München

Fabian.Trillsch@med. uni-muenchen.de

\title{
Ovarialkarzinom: älteren Patientinnen Chemotherapie nicht vorenthalten
}

\section{Zweifel, die zum Einsatz einer Chemotherapie bei älteren Patientinnen oft bestehen, sind nicht angebracht, wie aktuelle Studiendaten erneut zeigen.}

Ü ber die Hälfte aller epithelialen Ovarialkarzinome (EOC) wird bei Frauen über 65 Jahren diagnostiziert. Allerdings erhalten sie häufiger als Jüngere nur eine suboptimale Chemotherapie. Eine Erklärung hierfür könnten die Angst vor höherer Toxizität sowie mit dem Alter häufigere Komorbiditäten und Komedikationen sein. Im Gegensatz zu diesen Befürchtungen zeigte die Arbeitsgemeinschaft Gynäkologische Onkologie, dass eine Erstlinien-Chemotherapie mit Platin/Paclitaxel bei Frauen über 70 Jahren gut durchführbar und verträglich ist. Ziel der vorliegenden Studie war es, den Einfluss des Alters auf Toxizitäten vom Grad 3/4, die Notwendigkeit von Dosisreduktionen, ein vorzeitiges Ende der Chemotherapie sowie das progressionsfreie Überleben (PFS) bei Patientinnen mit re- zidiviertem EOC zu untersuchen. Es erfolgte eine Metaanalyse von 3 Phase-II/ III-Studien der Nordostdeutschen Gesellschaft für Gynäkologische Onkologie (TOWER, Topotecan Phase-III-Studie und Hector) mit 1.213 Patientinnen mit rezidiviertem EOC (Alter: median 59 Jahre). 349 Patientinnen waren $\geq 65$ Jahre alt; sie hatten deutlich häufiger kardiovaskuläre Erkrankungen und Diabetes als jüngere und nahmen deutlich häufiger Antihypertensiva ein. Hämatologische Toxizitäten wurden bei $58,9 \%$ der Patientinnen beobachtet, nicht-hämatologische bei $43,8 \%$. Hämatologische Toxizitäten waren häufiger bei über 65-Jährigen (Odds Ratio [OR] 1,35; $\mathrm{p}=0,028$ ), vor allem Anämien, Leukopenien und Thrombozytopenien. Dagegen traten nicht-hämatologische Toxizitäten in beiden Altersgruppen vergleichbar häufig auf $(p=0,901)$. Hinsichtlich der Anzahl der verabreichten Chemotherapiezyklen und der Notwendigkeit von Dosisreduktionen gab es keinen signifikanten Unterschied. Auch Therapieabbrüche wa- ren bei den älteren Patientinnen nicht häufiger. Es zeigte sich ein Trend zu einem kürzeren PFS bei älteren Patientinnen (Hazard Ratio [HR] 1,14; $\mathrm{p}=0,053$ ). Nach Adjustierung für kardiovaskuläre Erkrankungen und Diabetes ergab sich ein signifikant kürzeres PFS (HR 1,20; $\mathrm{p}=0,01)$ und auch Gesamtüberleben (HR 1,$21 ; \mathrm{p}=0,021$ ) bei den Älteren.

Fazit: Hämatologische Toxizitäten der Chemotherapie kommen bei EOC-Patientinnen über 65 Jahren öfter vor als bei jüngeren, haben aber keinen Einfluss auf die Häufigkeit von Therapieabbrüchen. Älteren Patientinnen sollte eine adäquate Chemotherapie allein wegen ihres Alters nicht vorenthalten werden. Daher sind in dieser Gruppe ein sorgfältiges geriatrisches Assessment und Monitoring erforderlich.

Judith Neumaier

Woopen $\mathrm{H}$ et al. Elderly ovarian cancer patients: An individual participant data meta-analysis of the North-Eastern German Society of Gynecological Oncology (NOGGO). Eur J Cancer.

2016;60:101-6. 